\title{
Guiding ab initio calculations by alchemical derivatives
}

M. to Baben', J. O. Achenbach, and O. A. von Lilienfeld'

Citation: The Journal of Chemical Physics 144, 104103 (2016); doi: 10.1063/1.4943372

View online: http://dx.doi.org/10.1063/1.4943372

View Table of Contents: http://aip.scitation.org/toc/jcp/144/10

Published by the American Institute of Physics

\section{Articles you may be interested in}

Fast and accurate predictions of covalent bonds in chemical space

The Journal of Chemical Physics 144, 174110174110 (2016); 10.1063/1.4947217

Communication: Understanding molecular representations in machine learning: The role of uniqueness and target similarity

The Journal of Chemical Physics 145, 161102161102 (2016); 10.1063/1.4964627

Electronic spectra from TDDFT and machine learning in chemical space

The Journal of Chemical Physics 143, 084111084111 (2015); 10.1063/1.4928757

Accurate ab initio energy gradients in chemical compound space

The Journal of Chemical Physics 131, 164102164102 (2009); 10.1063/1.3249969

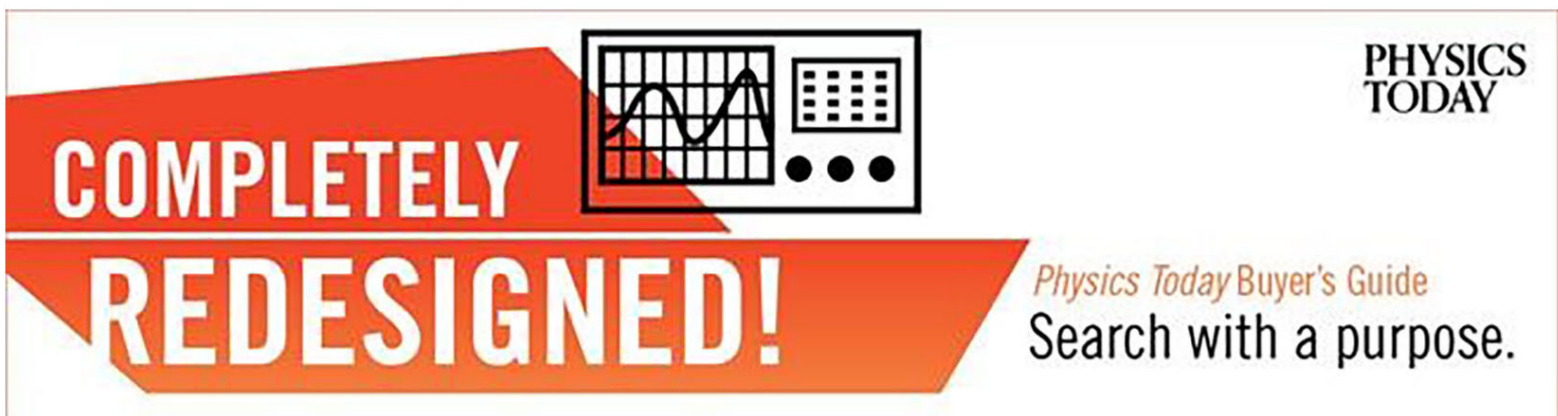




\title{
Guiding ab initio calculations by alchemical derivatives
}

\author{
M. to Baben, ${ }^{1,2,3, a)}$ J. O. Achenbach, ${ }^{2}$ and O. A. von Lilienfeld ${ }^{3,4,5, b)}$ \\ ${ }^{1}$ GTT Technologies, Kaiserstraße 100, 52134 Herzogenrath, Germany \\ ${ }^{2}$ Materials Chemistry, RWTH Aachen University, Kopernikusstr. 10, 52074 Aachen, Germany \\ ${ }^{3}$ Institute of Pure and Applied Mathematics, University of California Los Angeles, Los Angeles, \\ California 90095, USA \\ ${ }^{4}$ Institute of Physical Chemistry and National Center for Computational Design and Discovery of Novel \\ Materials (MARVEL), University of Basel, Klingelbergstrasse 80, 4056 Basel, Switzerland \\ ${ }^{5}$ General Chemistry (ALGC), Free University of Brussels, Pleinlaan 2, 1050 Brussels, Belgium
}

(Received 15 January 2016; accepted 24 February 2016; published online 10 March 2016)

\begin{abstract}
We assess the concept of alchemical transformations for predicting how a further and not-tested change in composition would change materials properties. This might help to guide ab initio calculations through multidimensional property-composition spaces. Equilibrium volumes, bulk moduli, and relative lattice stability of fcc and bcc $4 \mathrm{~d}$ transition metals $\mathrm{Zr}, \mathrm{Nb}, \mathrm{Mo}, \mathrm{Tc}, \mathrm{Ru}, \mathrm{Rh}, \mathrm{Pd}$, and $\mathrm{Ag}$ are calculated using density functional theory. Alchemical derivatives predict qualitative trends in lattice stability while equilibrium volumes and bulk moduli are predicted with less than $9 \%$ and $28 \%$ deviation, respectively. Predicted changes in equilibrium volume and bulk moduli for binary and ternary mixtures of $\mathrm{Rh}-\mathrm{Pd}-\mathrm{Ag}$ are in qualitative agreement even for predicted bulk modulus changes as large as $+100 \%$ or $-50 \%$. Based on these results, it is suggested that alchemical transformations could be meaningful for enhanced sampling in the context of virtual high-throughput materials screening projects. (C) 2016 AIP Publishing LLC. [http://dx.doi.org/10.1063/1.4943372]
\end{abstract}

\section{INTRODUCTION}

$A b$ initio calculations can be used to predict properties of hypothetical new materials, thereby reducing the number of experiments needed for materials discovery, ${ }^{1,2}$ e.g., compositions of hard-yet-ductile materials, ${ }^{3,4}$ new materials for Lithium-ion batteries, ${ }^{5}$ and materials with increased Seebeck coefficient for thermoelectric properties ${ }^{6}$ have been predicted based on quantum mechanical calculations and some predictions have already been validated experimentally. ${ }^{1,3,6,7}$

Many of these studies rely on a brute-force approach, where a large number of computationally demanding calculations are done to map composition-property space, ${ }^{2,4-6,8}$ as shown schematically in Fig. 1(a). This approach naturally consumes a lot of computational resources. Furthermore, from the 75 non-radioactive elements (ignoring noble gases), there are already more than $1 \times 10^{6}$ quaternary combinations possible, where in each system the individual content and crystal structure can be varied. This limits the prospects of success for brute-force methods to optimize materials' properties at reasonable cost. For organic chemistry, the number of small molecules alone has even been estimated to exceed $10^{60} .9$ To accelerate materials discovery, the alchemical potential has been proposed for a gradientsupported navigation through property-composition space, as shown in Fig. 1(b). ${ }^{10,11}$ The alchemical potential approximates the energy difference when transforming an element to an adjacent one by adding or removing a proton from the nucleus, e.g., changing $\mathrm{Pd}$ to $\mathrm{Rh}(\mathrm{Z}-1)$ or $\mathrm{Ag}(\mathrm{Z}+1) \cdot{ }^{10}$

\footnotetext{
a)to_baben@mch.rwth-aachen.de

b)anatole.vonlilienfeld@gmail.com
}

In the special case that the nuclei do not change position due to the alchemical transformation, the alchemical potential can be calculated by evaluating the effect of the surrounding electronic charge density on the energy of a test charge introduced at the nucleus. This number can easily be evaluated and is readily available, e.g., as standard output in the Vienna $A b$ initio Simulation Package (VASP). Other approaches such as derivatives with respect to coefficients in linear combinations of atomic potentials could have been used just as well. ${ }^{12}$ Alchemical transformations have been used successfully to describe the energy landscape of several molecular compounds. ${ }^{13-16}$ Sheppard et al. have shown that the alchemical potential can also be used to predict binding energies of oxygen to a $\mathrm{Pd}-\mathrm{Rh}-\mathrm{Ag}$ nanocluster from the simple calculation of a Pd nanocluster. ${ }^{17,18}$

However, only combinations of alchemical transformations have been considered with fixed geometry before and after transformation. Here, we show this boundary condition can be circumvented by calculating the alchemical potential for different volumes and structures. This is needed anyways to determine the ground state, and therefore not increasing the computational effort. Thus, equilibrium volumes, bulk moduli, and relative lattice stability of fcc and bec structures of $4 \mathrm{~d}$ transition metals $\mathrm{Zr}, \mathrm{Nb}, \mathrm{Mo}, \mathrm{Tc}, \mathrm{Ru}, \mathrm{Rh}, \mathrm{Pd}$, and $\mathrm{Ag}$ have been calculated using density functional theory (DFT) and predicted using alchemy. Additionally, equilibrium volume and bulk moduli for binary and ternary mixtures of $\mathrm{Rh}-\mathrm{Pd}-\mathrm{Ag}$ have been considered in order to test whether alchemical transformations can be used to design mixtures. From the comparison, it can be seen that trends of lattice stability, equilibrium volumes, and bulk modulus in chemical composition space can be predicted qualitatively. This 
a)

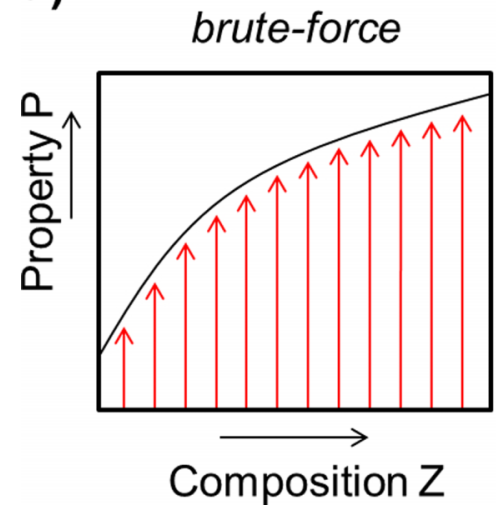

b)

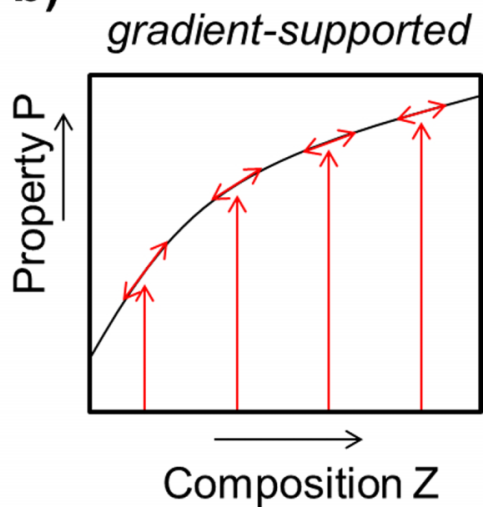

FIG. 1. (a) Brute-force approach for mapping property-composition space; (b) gradient-supported navigation through property-composition space. suggests that the alchemical potential can be used to guide $a b$ initio calculations leading to a more elegant and effective strategy for computational materials design with respect to the brute-force approach.

\section{THEORY}

Sheppard et al. have used an order parameter $\lambda$ with $0 \leq \lambda \leq 1$ to express the energy of a compound by linearization in chemical space. ${ }^{13,17}$ For $\lambda=0$, the energy is $E^{0}$, while for another compound $\lambda=1$ and the energy is approximated with the total differential,

$$
\begin{aligned}
E(\lambda=1) \cong & E^{0}+d E=E^{0}+\sum_{I} \mu_{n}\left(R_{\mathrm{I}}\right) \partial_{\lambda} N_{I} \\
& -\sum_{I} F_{I} \cdot \partial_{\lambda} R_{I}+\mu_{e} \partial_{\lambda} N_{e} .
\end{aligned}
$$

$\mu_{n}$ is the derivative of energy with respect to variation in nuclear charge, here labeled alchemical potential $\mu_{n}\left(R_{I}\right)$ since only changes of nuclear charge at position of atom $I$ are considered. $\partial_{\lambda} N_{\mathrm{I}}$ is the change in nuclear charge and takes the value of +1 or -1 here, depending on whether upward or downward alchemical transformations are considered. $F_{I}$ are the forces acting on atoms and $\mu_{e}$ is the change of energy due to change of number electrons $N_{e}$, a.k.a. electronic chemical potential. ${ }^{11,17}$ For pure elements in simple crystal structures, the forces acting on the atoms are equal to 0 due to symmetry; therefore, the third term can be neglected. For mixtures, however, atoms relax due to different bond lengths associated with bond between different elements. It will be shown later that equilibrium volume and bulk modulus predictions are in qualitative agreement, corroborating the assumption that $F_{I}$ is negligible compared to the other terms of Equation (1). $\mu_{e}$ is approximated as the Fermi energy $E_{f}$ as this is the lowest energy of an additional electron added to the material. ${ }^{18}$ Since $\mu_{n}$ and $\mu_{e}$ are standard output of $a b$ initio calculations, all variables in Equation (1) can readily be evaluated and it is possible to estimate the energy of the alchemically transformed material. In order to determine the ground state, $a b$ initio calculations have to be done at different volumes. Thus, energy-volume curves can be directly determined or alchemically derived and are fitted by the Birch-Murnaghan equation of state ${ }^{19}$ to derive equilibrium volume, ground state energy, and bulk modulus.

\section{CALCULATION DETAILS}

The ab initio calculations were done using the implementation of density functional theory ${ }^{20}$ in VASP. Generalizedgradient approximation was used with projector augmented wave potential. $^{21}$ Integration of the wave vectors in the reciprocal space was done with Monkhorst-Pack scheme ${ }^{22}$ and Blöchl-corrections were used within the tetrahedron method for Brillouin-zone integration. ${ }^{23}$ Additionally the cutoff energy for the calculations was set to $500 \mathrm{eV}$ with an electron relaxation convergence of $0.1 \mathrm{meV}$. For the calculations of fcc and bec Zr, Nb, Mo, Tc, Ru, Rh, Pd, Ag one unit cell was used with a k-point grid of $12 \times 12 \times 12$. The solid solutions in the $\mathrm{Rh}-\mathrm{Pd}-\mathrm{Ag}$ system were modelled as a $2 \times 2 \times 2$ fcc supercell with a k-point grid of $8 \times 8 \times 8$. Besides the pure elements, mixtures $\mathrm{M}_{0.5} \mathrm{M}_{0.5}^{\prime}$ and $\mathrm{M}_{0.5} \mathrm{M}_{0.25}^{\prime} \mathrm{M}^{\prime \prime}{ }_{0.25}\left(\mathrm{M}, \mathrm{M}^{\prime}, \mathrm{M}^{\prime \prime}=\mathrm{Rh}\right.$, $\mathrm{Pd}, \mathrm{Ag}$ ) were described as special quasirandom structures ${ }^{24}$ by minimizing the first three Warren-Cowley short-range order parameters ${ }^{25}$ using the locally self-consistent Green's function software package. ${ }^{26,27}$ Atoms were allowed to relax in the binary and ternary mixtures with a convergence criterion of $1 \mathrm{meV}$. All calculations were performed for non-magnetic configurations since these elements are not reported to exhibit spin polarized ground states. In fact, changes in spin polarization during the alchemical transformation are not yet considered in Equation (1) and an interesting field for further investigation.

\section{RESULTS AND DISCUSSION}

As an example, the directly calculated, i.e., using conventional DFT, and alchemically downward transformed elastic energies of fcc and bcc $\mathrm{Nb}$ are shown in Figure 2. For comparison, the elastic energies directly calculated for fcc and bcc $\mathrm{Zr}$ are included. The position of the energy difference $d E$ from Equation (1) is marked for two data points and can be as large as $0.6 \mathrm{eV} /$ atom. While the ground state of $\mathrm{Nb}$ is bcc with an energy difference between fcc and bcc of $\Delta E_{f c c-b c c}=0.33 \mathrm{eV} /$ atom the alchemically predicted energy difference of fcc and bcc $\mathrm{Zr}$ is $\Delta E_{f c c-b c c}^{a l c h-1}=-0.09 \mathrm{eV} /$ atom. Therefore, the ground state of $\mathrm{Zr}$ is correctly predicted, with only a small deviation to the directly calculated energy difference of $\Delta E_{f c c-b c c}=-0.04 \mathrm{eV} /$ atom. The alchemically predicted equilibrium volume of fcc $\mathrm{Zr}$ is $21.9 \AA^{3}$ /atom, 


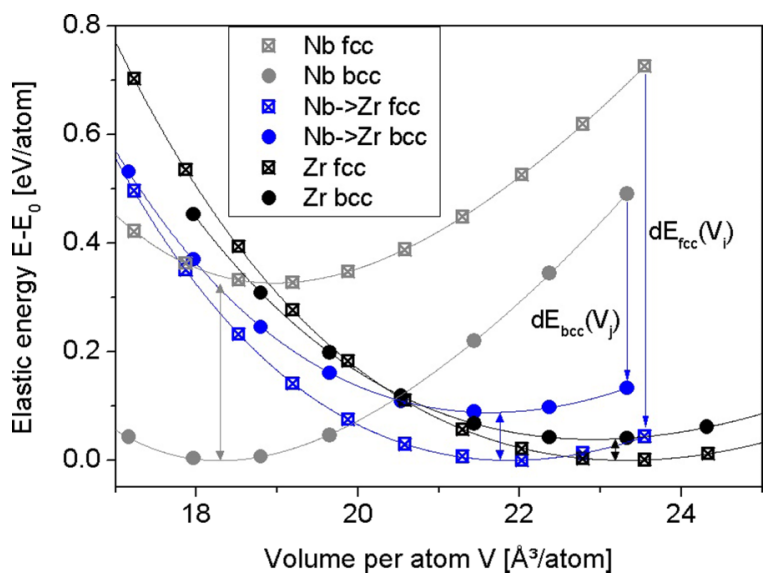

FIG. 2. Directly calculated and alchemically transformed elastic energies of fcc and bcc $\mathrm{Nb}$ and directly calculated elastic energy of fcc and bcc $\mathrm{Zr}$ as a function of volume. In all cases, the ground state energies are set to 0 for comparison. $\mathrm{dE}$ mark the energy changes during the alchemical transformation and the double-headed arrows mark the energy differences between the fcc and bcc phases.

while the directly calculated one is $23.3 \AA^{3}$ /atom, indicating an error of $6 \%$, which is similar to the accuracy of ab initio calculations of $3 \%-6 \% .{ }^{28}$ It is noteworthy that the alchemically predicted volume change when fcc $\mathrm{Nb}$ is transformed to fcc $\mathrm{Zr}$ is $+16 \%$ while the observed change is $+23 \%$ and thus in very good qualitative agreement, which is a necessary condition to use alchemical transformations to navigate through propertycomposition space as shown in Fig. 1(b).

The directly calculated and alchemically transformed energy differences between fcc and bcc elements, of $\Delta E_{f c c-b c c}$ and $\Delta E_{f c c-b c c}^{a l c h+/-1}$, are shown in Fig. 3. It should be noted that two alchemical transformations are possible, i.e., the upward $(\mathrm{Zr} \rightarrow \mathrm{Nb})$ and the downward $(\mathrm{Nb} \rightarrow \mathrm{Zr})$ transformation $\Delta E_{f c c-b c c}^{a l c h l}$ and $\Delta E_{f c c-b c c}^{a l c h}$. For comparison, data reported by Ozolinsš and Körling using full-potential GGA calculations ${ }^{29}$ and data obtained by the CALPHAD (CALculation of

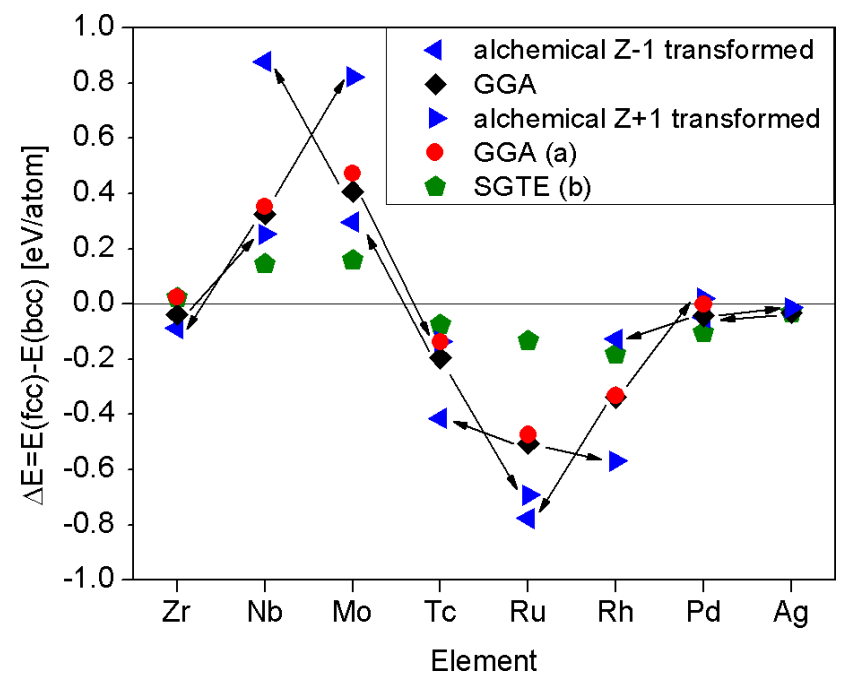

FIG. 3. Directly calculated (black diamond) and alchemically transformed (downward $(\mathrm{Z}-1)$ and upward $(\mathrm{Z}+1)$ ) energy difference between pure elements in fcc and bcc structures. (a) Full-potential GGA data, ${ }^{29}$ (b) SGTE data. $^{30}$
PHAse Diagrams) method from the SGTE (Scientific Group Thermodata Europe) database ${ }^{30}$ are included.

Generally, good agreement between predicted and directly calculated lattice stabilities $\Delta \mathrm{E}$ (fcc-bcc) is observed. Notable exceptions with errors of $0.55 \mathrm{eV} /$ atom and 0.41 $\mathrm{eV} /$ atom, respectively, are the alchemical transformations from $\mathrm{Mo}$ to $\mathrm{Nb}$ and vice versa. This might be due to the fact that Mo is the element with the maximum $\Delta \mathrm{E}$ (fcc-bcc), so that the energy-chemical compound space relation exhibits a strong bowing while according to Equation (1) only a linear expansion of energy in chemical compound space is taken into account and higher order terms are ignored. Nevertheless, it should be noted that the predictions of relative stability of fcc and bcc are also qualitatively in agreement with both reported full-potential GGA calculated data as well as experimentally derived data in the SGTE database, indicating that alchemical transformations and the theory proposed here can be used to guide $a b$ initio calculations of lattice stability.

To further study what properties can be predicted by alchemical transformations, results for the atomic volume and bulk modulus are shown in Figures 4 and 5, respectively.

While the general trend of volume with changing elements is well reproduced, the volume that is predicted by a downward (upward) transformation is 6\%-8\% smaller (2\%-6\% larger) than the directly calculated volume. It should be noted that the accuracy of DFT calculations of equilibrium volume is typically $\sim 6 \%{ }^{28}$ and the prediction is therefore at least within $14 \%$ deviation. Where available, calculated data using full-potential GGA calculations, that are themselves within $4 \%$ deviation of experimental data, ${ }^{29}$ are included and show excellent agreement with our calculated data.

Since the difference of the here calculated bulk modulus of fcc and bcc metals is less than $10 \%$, the bulk modulus calculated using full-potential GGA is included in Fig. 5 irrespective of crystal structure.

Very good agreement with deviations smaller than $28 \%$ is observed for the bulk modulus prediction, even though the difference in bulk modulus between elements next to each other in the periodic table of elements

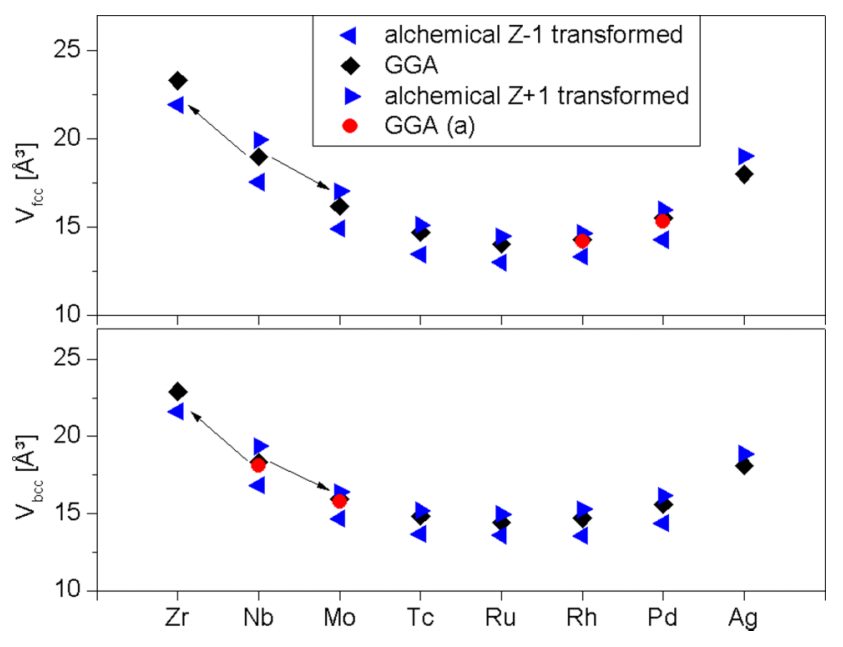

FIG. 4. Directly calculated and alchemically transformed volume for fcc (top) and bcc (bottom). Arrows indicate the alchemical transformations. (a) Full-potential GGA data ${ }^{29}$ shown for comparison. 


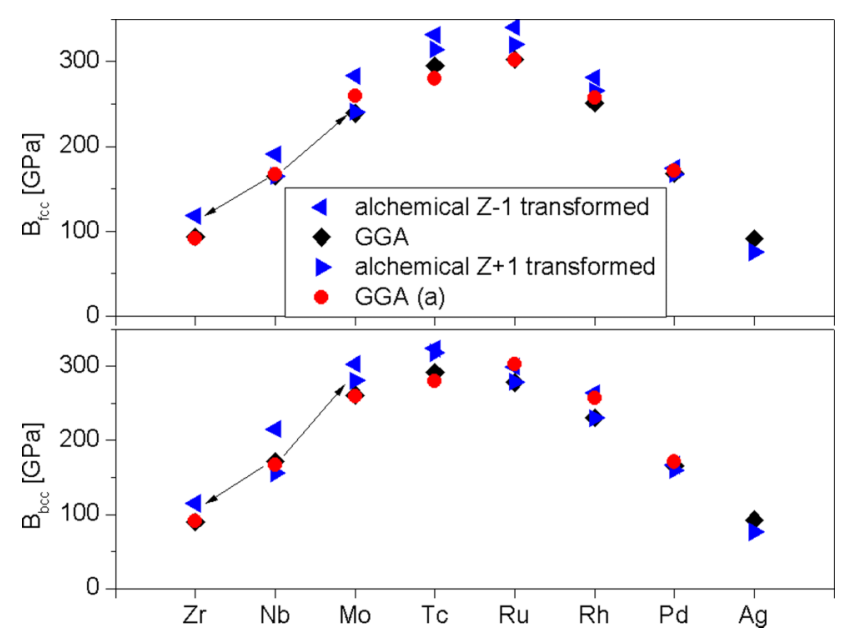

FIG. 5. Directly calculated and alchemically transformed volume for fcc (top) and bec (bottom). (a) Full-potential GGA data ${ }^{29}$ shown for comparison.

can be up to $80 \%(\mathrm{Zr} \rightarrow \mathrm{Nb}, \mathrm{Pd} \rightarrow \mathrm{Rh}$ ), indicating the excellent potential of alchemical transformations to guide $a b$ initio calculations for materials design. The bulk moduli are more often overestimated than underestimated and the alchemical downward transformation generally yields a larger overestimation than the upward transformation. This might be due to the fact that volume is stronger underestimated for alchemical downward transformations than it is overestimated for upward transformations. The reasons for the systematic differences between upward and downward transformations are not clear at this point.

For meaningful navigation of property-composition space, it is very desirable to be able to predict properties of mixtures. Therefore, equilibrium volume and bulk modulus are calculated and predicted for fcc $\mathrm{M}, \mathrm{M}_{0.5} \mathrm{M}_{0.5}^{\prime}$, and $\mathrm{M}_{0.5} \mathrm{M}_{0.25}^{\prime} \mathrm{M}^{\prime \prime}{ }_{0.25}$, where $\mathrm{M}, \mathrm{M}^{\prime}$, and $\mathrm{M}^{\prime \prime}$ are $\mathrm{Rh}$, $\mathrm{Pd}$, or $\mathrm{Ag}$. The relative equilibrium volume change $V($ transformed $) / V$ (original) and bulk modulus change $B($ transformed)/B(original) are shown in Figures 6 and 7, where the directly calculated ratio is plotted as a function of the predicted ratio. It can be seen that the alchemically predicted

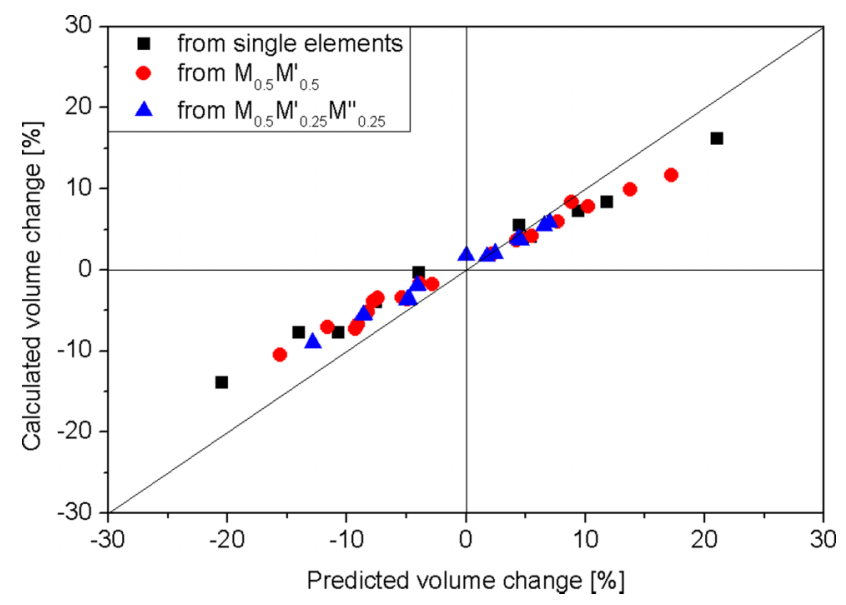

FIG. 6. Calculated and predicted volume change from calculated single elements $\mathrm{M}$, from binary mixtures $\mathrm{M}_{0.5} \mathrm{M}_{0.5}^{\prime}$ and from ternary mixtures $\mathrm{M}_{0.5} \mathrm{M}_{0.25}^{\prime} \mathrm{M}^{\prime \prime}{ }_{0.25}$, where $\mathrm{M}, \mathrm{M}^{\prime}$, and $\mathrm{M}^{\prime \prime}$ are $\mathrm{Rh}, \mathrm{Pd}$, or $\mathrm{Ag}$.

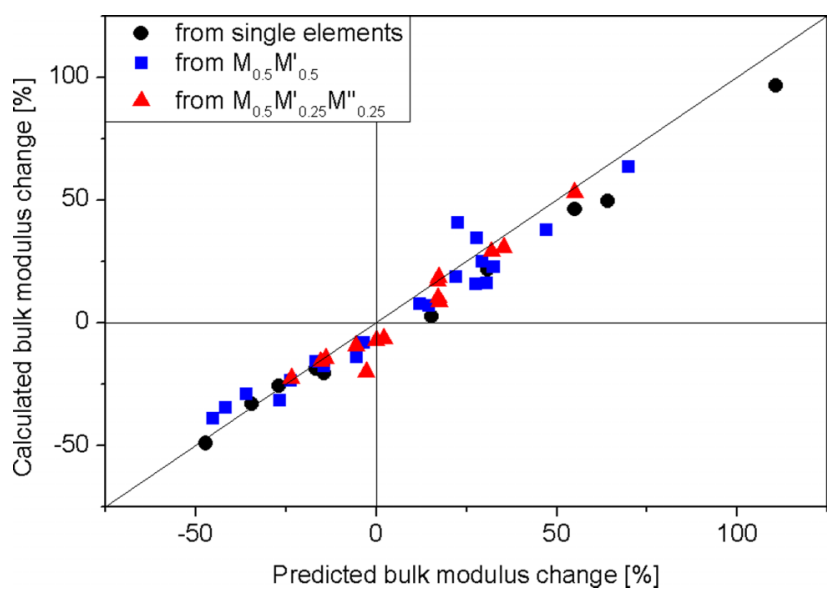

FIG. 7. Calculated and predicted bulk modulus change from calculated single elements $\mathrm{M}$, from binary mixtures $\mathrm{M}_{0.5} \mathrm{M}_{0.5}^{\prime}$ and from ternary mixtures $\mathrm{M}_{0.5} \mathrm{M}_{0.25}^{\prime} \mathrm{M}_{0.25}^{\prime \prime}$, where $\mathrm{M}, \mathrm{M}^{\prime}$, and $\mathrm{M}^{\prime \prime}$ are $\mathrm{Rh}$, $\mathrm{Pd}$, or $\mathrm{Ag}$.

changes in volume and bulk modulus are qualitatively correct for nearly all systems. Here, qualitatively means that for a predicted increase (decrease) the calculated change shows an increase (decrease) as well. The slopes of the linear fits of the data points in Figures 6 and 7 indicate that alchemical transformations overestimate the changes systematically. This is not critical for materials design since the trend is correctly reproduced. From the data shown in Figures 6 and 7, there are two aspects that are noteworthy: First, even for predicted bulk modulus changes as large as $+100 \%$ or $-50 \%$, the prediction is still in good agreement with the directly calculated change, showing that even extreme differences in bulk modulus can be correctly estimated. Second, the single calculation of $\mathrm{Pd}$ can be transformed into $\mathrm{Rh}, \mathrm{Ag}, \mathrm{Rh}_{0.5} \mathrm{Pd}_{0.5}, \mathrm{Pd}_{0.5} \mathrm{Ag}_{0.5}$, $\mathrm{Rh}_{0.5} \mathrm{Pd}_{0.25} \mathrm{Ag}_{0.25}$, or $\mathrm{Rh}_{0.25} \mathrm{Pd}_{0.25} \mathrm{Ag}_{0.5}$ which shows that multi-dimensional navigation in chemical compound space is possible by alchemical transformations.

\section{CONCLUSION}

We have shown that alchemical transformations can be used to navigate the multidimensional property-composition space of solid transition metals. The boundary condition of fixed geometry before and after alchemical transformation can be circumvented by calculating the alchemical potential for different volumes and structures. This is anyways needed to determine the ground state and therefore not increasing the computational effort. Equilibrium volumes, bulk moduli, and relative lattice stability of fcc and bec $4 \mathrm{~d}$ transition metals $\mathrm{Zr}$, $\mathrm{Nb}, \mathrm{Mo}, \mathrm{Tc}, \mathrm{Ru}, \mathrm{Rh}, \mathrm{Pd}$, and Ag have been calculated and predicted. From the comparison, one can see that trends in lattice stability of the $4 \mathrm{~d}$ transition metals can be predicted qualitatively, and equilibrium volumes and bulk moduli are predicted with less than $9 \%$ and $28 \%$ deviation, respectively. Additionally, changes in equilibrium volume and bulk moduli for binary and ternary mixtures of $\mathrm{Rh}-\mathrm{Pd}-\mathrm{Ag}$ are predicted qualitatively correct. Based on these results, we conclude that alchemical transformations could be used to enhance high-throughput materials genome initiative projects, such as the materials project, ${ }^{31}$ to predict how a further and not-tested 
change in composition would affect properties that can be derived from the total energy of the system.

\section{ACKNOWLEDGMENTS}

M.t.B. acknowledges financial support by RWTH Aachen University (Start-Up grant). Simulations were performed partly with computing resources granted by JARA-HPC from RWTH Aachen University under Project No. JARA0131. O.A.v.L. acknowledges support from the Swiss National Science Foundation (No. PP00P2_138932). M.t.B. and O.A.v.L. acknowledge support from the long program "Navigating Chemical Compound Space for Materials and Bio Design,” IPAM, UCLA.

${ }^{1}$ J. K. Norskov, T. Bligaard, J. Rossmeisl, and C. H. Christensen, Nat. Chem. 1, 37 (2009).

${ }^{2}$ S. Curtarolo, G. L. W. Hart, M. B. Nardelli, N. Mingo, S. Sanvito, and O. Levy, Nat. Mater. 12, 191 (2013).

${ }^{3}$ J. Emmerlich, D. Music, M. Braun, P. Fayek, F. Munnik, and J. M. Schneider, J. Phys. D: Appl. Phys. 42, 6 (2009).

${ }^{4}$ D. G. Sangiovanni, L. Hultman, and V. Chirita, Acta Mater. 59, 2121 (2011).

${ }^{5}$ G. Hautier, A. Jain, S. P. Ong, B. Kang, C. Moore, R. Doe, and G. Ceder, Chem. Mater. 23, 3495 (2011).

${ }^{6}$ D. Music, Y.-T. Chen, R. W. Geyer, P. Bliem, and J. M. Schneider, Mater. Res. Express 1, 045034 (2014).

${ }^{7}$ H. Kindlund, D. G. Sangiovanni, L. Martínez-de-Olcoz, J. Lu, J. Jensen, J. Birch, I. Petrov, J. E. Greene, V. Chirita, and L. Hultman, APL Mater. 1, 042104 (2013).

${ }^{8}$ M. de Jong, W. Chen, T. Angsten, A. Jain, R. Notestine, A. Gamst, M. Sluiter, C. Krishna Ande, S. van der Zwaag, J. J. Plata, C. Toher, S. Curtarolo, G. Ceder, K. A. Persson, and M. Asta, Sci. Data 2, 150009 (2015).
${ }^{9}$ P. Kirkpatrick and C. Ellis, Nature 432, 823 (2004).

${ }^{10}$ O. A. von Lilienfeld, R. D. Lins, and U. Rothlisberger, Phys. Rev. Lett. 95, 153002 (2005).

${ }^{11}$ O. A. von Lilienfeld and M. E. Tuckerman, J. Chem. Phys. 125, 154104 (2006).

${ }^{12}$ M. Wang, X. Hu, D. N. Beratan, and W. Yang, J. Am. Chem. Soc. 128, 3228 (2006).

${ }^{13}$ O. A. von Lilienfeld, J. Chem. Phys. 131, 164102 (2009).

${ }^{14}$ O. A. von Lilienfeld, Int. J. Quantum Chem. 113, 1676 (2013).

${ }^{15}$ R. Balawender, M. A. Welearegay, M. Lesiuk, F. De Proft, and P. Geerlings, J. Chem. Theory Comput. 9, 5327 (2013).

${ }^{16}$ K. Y. S. Chang, S. Fias, R. Ramakrishnan, and O. A. von Lilienfeld, e-print arXiv:1509.02847 (2015).

${ }^{17}$ D. Sheppard, G. Henkelman, and O. A. von Lilienfeld, J. Chem. Phys. 133, 084104 (2010).

${ }^{18}$ D. Sheppard, Ph.D. thesis, University of Texas at Austin, 2010.

${ }^{19}$ F. Birch, J. Geophys. Res. 83, 1257, doi:10.1029/JB083iB03p01257 (1978).

${ }^{20}$ W. Kohn and L. J. Sham, Phys. Rev. 140, 1133 (1965).

${ }^{21}$ G. Kresse and D. Joubert, Phys. Rev. B 59, 1758 (1999).

${ }^{22}$ H. J. Monkhorst and J. D. Pack, Phys. Rev. B 13, 5188 (1976).

${ }^{23}$ P. E. Blöchl, O. Jepsen, and O. K. Andersen, Phys. Rev. B 49, 16223 (1994).

${ }^{24}$ A. Zunger, S. H. Wei, L. G. Ferreira, and J. E. Bernard, Phys. Rev. Lett. 65, 353 (1990).

${ }^{25}$ J. M. Cowley, J. Appl. Phys. 21, 24 (1950).

${ }^{26}$ I. A. Abrikosov, A. M. N. Niklasson, S. I. Simak, B. Johansson, A. V. Ruban, and H. L. Skriver, Phys. Rev. Lett. 76, 4203 (1996).

${ }^{27}$ I. A. Abrikosov, S. I. Simak, B. Johansson, A. V. Ruban, and H. L. Skriver, Phys. Rev. B 56, 9319 (1997).

${ }^{28} \mathrm{O}$. Eriksson, Encyclopedia of Materials: Science and Technology (Elsevier, 2006), p. 1.

${ }^{29}$ V. Ozolins and M. Korling, Phys. Rev. B 48, 18304 (1993).

${ }^{30}$ A. T. Dinsdale, CALPHAD: Comput. Coupling Phase Diagrams Thermochem. 15, 317 (1991).

${ }^{31}$ A. Jain, S. P. Ong, G. Hautier, W. Chen, W. D. Richards, S. Dacek, S. Cholia, D. Gunter, D. Skinner, G. Ceder, and K. A. Persson, APL Mater. 1, 011002 (2013). 